\title{
Asymptotic method and numerical analysis for self-excited vibration in rolling mill with clearance
}

\author{
Hongguang $\mathrm{Li}^{\mathrm{a}}$, Bangchun Wen ${ }^{\mathrm{b}}$ and \\ Jianwu Zhang ${ }^{\mathrm{a}}$ \\ ${ }^{a}$ School of Mechanical Engineering, Shanghai Jiao \\ Tong University, Shanghai, 200030, China \\ ${ }^{\mathrm{b}}$ School of Mechanical Engineering, Northeastern \\ University, Shenyang, 110006, P.R. China
}

Received 21 April 1999

Revised 3 September 2000

In this paper, a dynamic model is proposed for analysis of nonlinear vibrations of rolling mills with fixed and time-varying clearances. Self-excited vibrations of the system that is basically involved with piece-wise nonlinearity and discontinuities are investigated via asymptotic method. It is shown by numerical results obtained for the nonlinear system with a time-varying clearance that different forms of nonlinear vibrations appear to be periodic, quasi-periodic and chaotic. Influence of the system parameters on the nonlinear vibration behaviors is examined by applying the Poincare sections, the bifurcation diagram and the largest Lyapunov exponent. New phenomena are observed in nonlinear motions of the rolling mill mechanism and are of significant importance for design of this type of mechanical systems.

\section{Introduction}

In modern rolling mills, the rolling power and speed are gradually increased. Although the rolling mills are designed with larger safety margin, some mechanical defaults caused due to torsional vibrations still exist. As the rolling mills work always with unavoidable slippages between roll and workpiece, large oscillations are possibly encountered and dangerous to the equipments. During that time, the rolling mill, motor, foundation and then whole building are obviously rocked. Dynamic interactions between the roll and the workpiece influence directly both quality and stability of the material processing technology. The overall interactive factors of the rolling mill including ferric oxides attached to the surface of steel at rolling and some nonlinear problems of the rolling mill system are studied.

Vibrations of the rolling mill caused by decreasing coefficient of friction between the roller and the steel was studied by Chen [3]. A simple model of the rolling mill with fixed clearances is presented by $\mathrm{Xu}[9,10]$, using Poincare-Bendixson theorem. It is shown that the self-excited vibrations in the rolling mill is governed by a generalized Lienard function and the corresponding limit cycle in the phase portraits turns out to be stable. Recent investigations to nonlinear vibration have been made by Guckenhimer and Holmes [5], Thompson et al. [7], Moon et al. [6] and Chen [2], pointing out importance of chaotic motions to many real applications. Some engineering problems with clearance have been analyzed, e.g. the machine tool-cutting process system with clearance by Wiercigroch [8], rub rotor system by Zhu [11]. However, most investigations considered the dynamic systems with a fixed clearance.

The present research is intended to establish a simple dynamic model for the rolling mill with fixed and timevarying clearances, and further to simulate a variety of dynamic responses of the nonlinear system undergoing periodic, quasi-periodic and chaotic vibrations. A new asymptotic solution is constructed to the initial value problems based upon the theory of Bogoliubov et al. [1], and numerical results are presented to gain a clear insight into the origin of chaotic motions in the rolling mill system.

\section{Equations of motion}

The governed differential equation about rolling mill can be written as

$$
\frac{\partial^{2} X}{\partial t^{2}}=f\left(t, X, \frac{\partial X}{\partial t}, P\right)
$$

where $X$ is displacement vector; $P$ is parameters vector; $f$ is forces vector depended on internal and exter- 


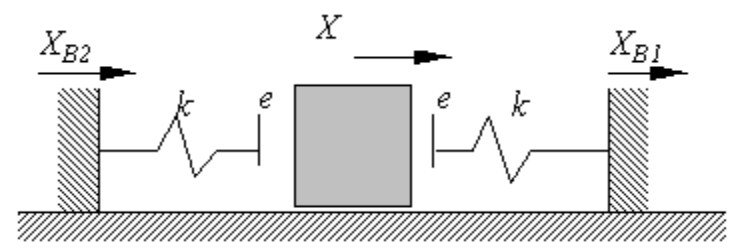

Fig. 1. A simplified model of the rolling mill.

nal excitations. In this paper, $f$ is consisted of two parts: $f(t, X)$ is the restoring force, and $f(\dot{X})$ is the frictional force. Based on a SDOF model provided by $\mathrm{Xu}$ [9], considering the effects of elastic oscillation of driving system, unstable rotation of the rotor and so on, we assume the boundaries of the model are moveable, and illustrate a modified mechanical model in Fig. 1, therefore $f(t, X)$ and $f(\dot{X})$ can be written as follows

$$
\begin{aligned}
& \begin{cases}f(t, X)=\omega^{2}\left(X-e-X_{B 1}\right) & X>e+X_{B 1} \\
f(t, X)=0 & -\left(e-X_{B 2}\right) \leq X \leq e+X_{B 1} \\
f(t, X)=\omega^{2}\left(X+e-X_{B 2}\right) & X<-\left(e-X_{B 2}\right)\end{cases} \\
& f(\dot{X})=-\alpha \dot{X}+\beta \dot{X}^{3}
\end{aligned}
$$

the parameters of the system are expressed as

$$
\omega^{2}=\frac{k}{J} \quad \alpha=\frac{\left(\frac{D}{2}\right)^{2} N c}{J} \quad \beta=\frac{\left(\frac{D}{2}\right)^{4} N d}{J}
$$

where $c$ and $d$ are constants, $c \in(0.03 \sim 0.09)$ and $d \in(0.0015 \sim 0.0033)$ respectively, $D$ is the diameter of roll, $N$ is press, $J$ is rotating inertia of rotor, and $k$ is the rotating stiffness of driving system $[3,9]$. Considering the equilibrium of moments with fixed clearances, $X_{B 1}=X_{B 2}=0$, a differential equation can be derived of the following form as

$$
\ddot{X}+g(X)-\alpha \dot{X}+\beta \dot{X}^{3}=0
$$

where $g(X)$ is a piece-wise linear restoring force, that is

$$
g(X)= \begin{cases}\omega^{2}(X-e) & X>e \\ 0 & -e \leqslant X \leqslant e \\ \omega^{2}(X+e) & X<-e\end{cases}
$$

with the clearances as illustrated in Fig. 2.

The motion of the system with fixed clearances is governed by a nonlinear differential equation of the second order.

\section{Asymptotic solution}

The equation (3) can be rewritten in the following form as

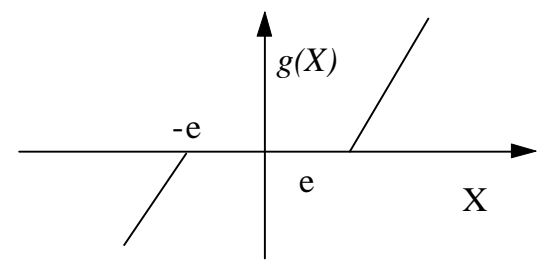

Fig. 2. Expression of piece-wise restoring force.

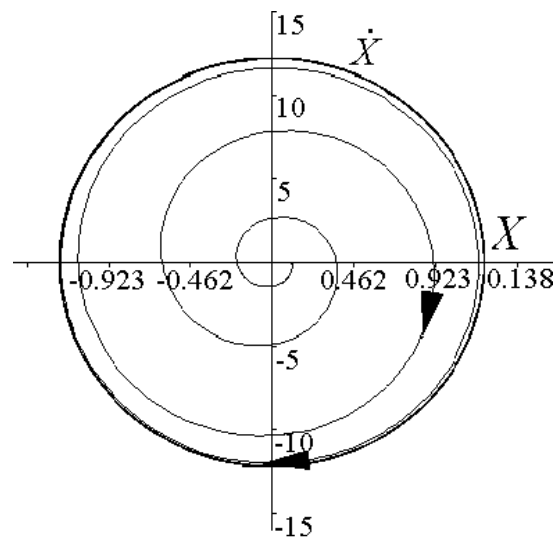

Fig. 3. Phase portrait of the system with a fixed clearance $e=0.001$ via asymptotic solution.

$$
\ddot{X}+\omega^{2} X=h(X)+\alpha \dot{X}-\beta \dot{X}^{3}
$$

where

$$
h(X)= \begin{cases}\omega^{2} e & X>e \\ \omega^{2} X & X \leqslant|e| \\ -\omega^{2} e & X<-e\end{cases}
$$

According to the asymptotic method developed by Bogoliubov et al. [1], the first approximation of the equation (4) can be expressed as

$$
X=a(t) \cos \psi(t)
$$

Substitution of equation (5) into the right side of equation (4) results in

$$
\begin{aligned}
\ddot{X}+\omega^{2} X= & h(a \cos \psi)-\alpha \cdot a \omega \sin \psi \\
& +\beta(a \omega \sin \psi)^{3}
\end{aligned}
$$

in which

$$
h(a \cos \psi)= \begin{cases}\omega^{2} e & -\theta_{e}>\psi>\theta_{e} \\ \omega^{2} a \cos \psi & \theta_{e} \leqslant \psi \leqslant \pi-\theta_{e} \\ & \pi+\theta_{e} \leqslant \psi \leqslant 2 \pi-\theta_{e} \\ -\omega^{2} e & \pi-\theta_{e}<\psi<\pi+\theta_{e}\end{cases}
$$

and $\theta_{e}=\arccos (e / a)$. Refering to the nonlinear term on the right side of Eq. (6), the corresponding nonlinear function is defined below 


$$
\begin{gathered}
\varepsilon \dot{f}(-a \omega \sin \psi)=h(a \cos \psi)-\alpha \dot{a} \omega \sin \psi \\
\beta(a \omega \sin \psi)^{3}
\end{gathered}
$$

where $\varepsilon$ is a small positive parameter. The differential functions of both amplitude and phase presented as following

$$
\begin{aligned}
\frac{d a}{d t}= & -\frac{\varepsilon}{2 \pi \omega} \int_{0}^{2 \pi} f(-a \omega \sin \psi) \cdot \sin \psi \cdot d \psi \\
= & \frac{\alpha}{2} a-\frac{3 \beta}{8} a^{3} \omega^{2} \\
\frac{d \psi}{d t}= & \omega-\frac{\varepsilon}{2 \pi a \omega} \\
& \int_{0}^{2 \pi} f(-a \omega \sin \psi) \cdot \cos \psi \cdot d \psi \\
= & \omega-\frac{\omega}{2 \pi a} \\
& {\left[4 e \sin \theta_{e}+a\left(\pi-2 \theta_{e}+2 \frac{e}{a} \sin \theta_{e}\right)\right] }
\end{aligned}
$$

Integration of the equation (8) and imposition of the initial value $a=a_{0}$ at $t=0$ lead to the approximate solution for the amplitude of vibration

$$
a=\sqrt{\frac{\alpha \cdot a_{0}^{2} e^{\alpha t}}{\alpha-\frac{3 \beta}{4} \omega^{2} a_{0}^{2}+\frac{3 \beta}{4} \omega^{2} a_{0}^{2} e^{\alpha t}}}
$$

Clearly, the amplitude of self-excited vibration in the rolling mill increasingly varies with time, and approaches to the maximum of the time limit

$$
\alpha_{\infty}=\lim _{t \rightarrow \infty} a(t)=\sqrt{\frac{4 \alpha}{3 \beta \omega^{2}}}
$$

In fact, the amplitude $a_{\infty}$ is stable for the self-excited vibration in the rolling mill. The overall amplitude is equal to the amplitude of equation (11) and that of the static force $\omega^{2} e$

$$
A=a_{\infty}+e=\sqrt{4 \alpha / 3 \beta \omega^{2}}+e
$$

substitution of Eq. (10) into Eq. (9) with considering that $e \ll a, \sin \theta_{e} \approx 1$, and yields the following equation as

$$
\frac{d \psi}{d t}=\omega-\frac{3 \omega e}{\pi a}
$$

As seen from the above equation (13), the frequency of the self-excited vibration system is a function of time but no longer a constant.

The solution to the equation governing self-excited vibrations in rolling mill may be obtained. Equation (13) is valid when the parameter $e$ is very small in the first approximation. In Fig. 3, phase portrait of the system (6) by the asymptotic technique is plotted. Taking a numerical example for the selfexcited vibration system with the specific parameters $\omega=102.5, \alpha=42.766, \beta=0.383$, and $e=0.001$.

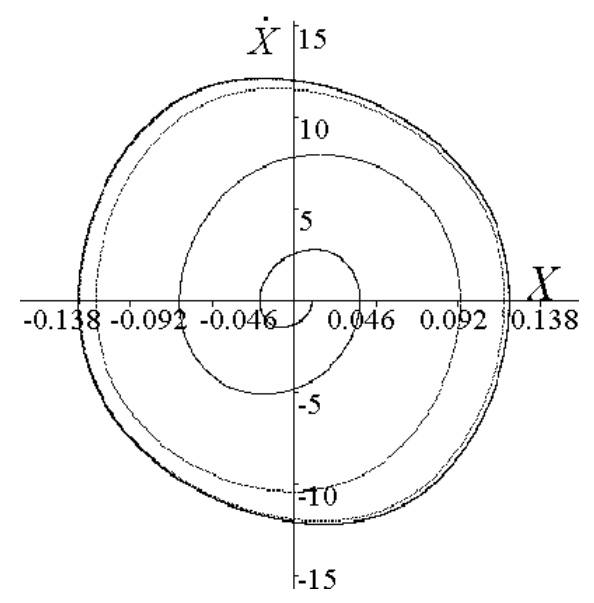

Fig. 4. Phase portrait of the system with a fixed clearance $e=0.001$ via numerical solution.

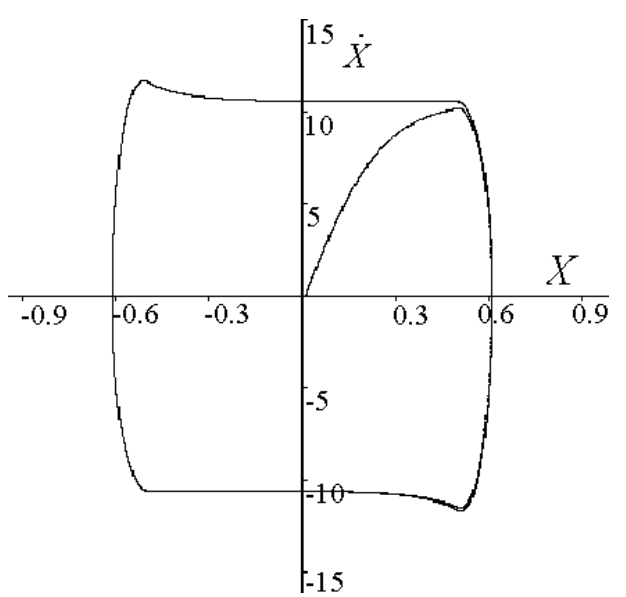

Fig. 5. Phase portrait of the system with a fixed clearance $e=0.5$ via numerical solution.

\section{Numerical analysis}

An extensive analysis can be performed by numerical solution. By setting $\dot{X}=y$ in Eq. (3), the differential equation of the second order can be transformed to the state equations of form as

$$
\left\{\begin{array}{l}
\dot{X}=y \\
\dot{y}=\alpha \cdot y-\beta \cdot y^{3}-g(X)
\end{array}\right.
$$

where the restoring force $g(X)$ is treated as piecewise like the one defined previously. The modified Runge-Kutta algorithm of the fourth order developed by Gear [4] is used for numerical solution of the initial value problem in Eq. (14). 


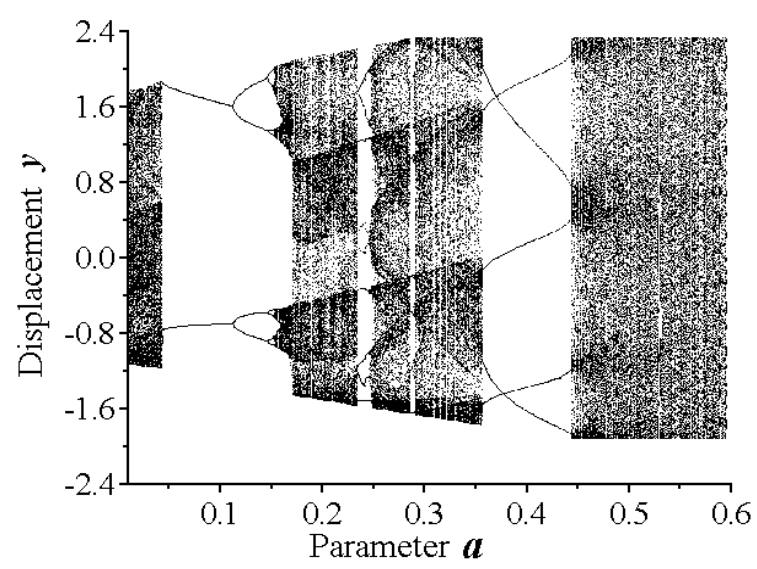

Fig. 6. Bifurcation diagram of the nonlinear system with a time-varying clearance.

\subsection{The rolling mill with a fixed clearance}

Numerical analysis of self-excited vibrations of the rolling mill with a fixed clearance is carried out. As known in Eq. (14), The dynamic system is nonlinear and has a piece-wise linear restoring force. Small increments of time are still required in the Runge-Kutta algorithm. In each of iteration, the displacement $X$ may be predicted, because the restoring force $g(X)$ is composed of three folded lines described by linear functions, respectively.

Numerical results for the phase plane trajectory of the system (14) with a fixed clearance is illustrated in Fig. 4. From comparison between the two phase portraits in Fig. 3 and Fig. 4, it is demonstrated that both trajectories are similar. Here, parameters required are provided $\omega=102.5, \alpha=12.06 / 0.282, \beta=0.108 / 0.282$, and $e=0.001$.

The trajectories in the Fig. 3 and Fig. 4 are in closed agreement with each other, which are stable limit cycles. The self-excited vibrations of the system are also stable, and this coincides with the conclusion of the asymptotic analysis. For examination of the clearance effect on motions of the system, assumably taking the clearance $e=0.5$, numerical predictions computed by the Runge-Kutta method are shown in Fig. 5 for the phase plane trajectory.

The trajectory of the system with the clearance $e=$ 0.5 are quite different from that of the system with the clearance $e=0.001$. In the case that the clearance is broad enough, the velocity will be stable

$$
\dot{\varphi}=\sqrt{\frac{\beta}{\alpha}}
$$

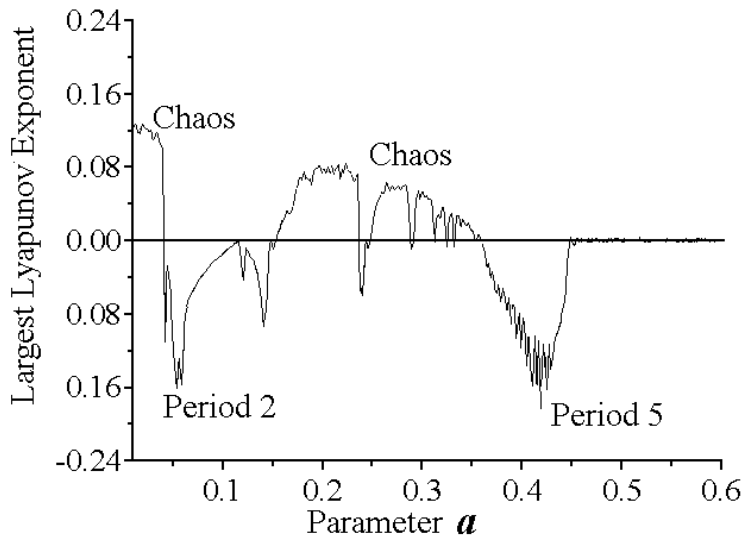

Fig. 7. The largest Lyapunov exponents for $b=0.3, \varpi=1.1$ and $\gamma=0.5$.

\subsection{The rolling mill with time-varied clearance}

Numerical analysis of the model with a time-varying clearance is also undertaken, in particular, for the purpose of exploring nonlinear dynamic behaviors of the system with inclusion of movable boundaries. In fact, the clearance becomes changeable with time, since elastic torsional oscillation of the driving system, and unstable rotational speed of the rotor cause complicated motions of the boundaries. For simplicity, it is assumed in the dynamic system that vibrations of the two boundaries are periodic, and are expressible as

$$
X_{B 1}=X_{B 2}=\delta \sin \omega_{B} t
$$

Thus, the restoring forces can be written in piecewise form as follows

$$
\begin{cases}f(t, X)=\omega^{2}\left(X-e-\delta \sin \omega_{B} t\right) & X>e+\delta \sin \omega_{B} t \\ f(t, X)=0 & -\left(e-\delta \sin \omega_{B} t^{t)} \leq X\right. \\ & \leq e+\delta \sin \omega_{B} t \\ f(t, X)=\omega^{2}\left(X+e-\delta \sin \omega_{B} t\right) & X<-\left(e-\delta \sin \omega_{B} t\right)\end{cases}
$$

Therefore, the differential equation of the dynamic model can be presented below

$$
\ddot{X}+g(t, X)-\alpha \dot{X}+\beta \dot{X}^{3}=0
$$

where $g(t, X)=f(t, X)$. Introduction of nondimensional variables and parameters

$$
\begin{aligned}
& \omega t=\tau \quad \frac{X}{e}=y \quad a=\frac{\alpha}{\omega} \\
& b=\beta \cdot \omega \cdot e^{2} \quad \bar{\omega}=\frac{\omega_{B}}{\omega} \quad \gamma=\frac{\delta}{e}
\end{aligned}
$$

and insertion to the governing equation (18) result in the equation of the non-dimensional form as

$$
y^{\prime \prime}+h(\tau, y)-a y^{\prime}+b y^{\prime 3}=0
$$

where 


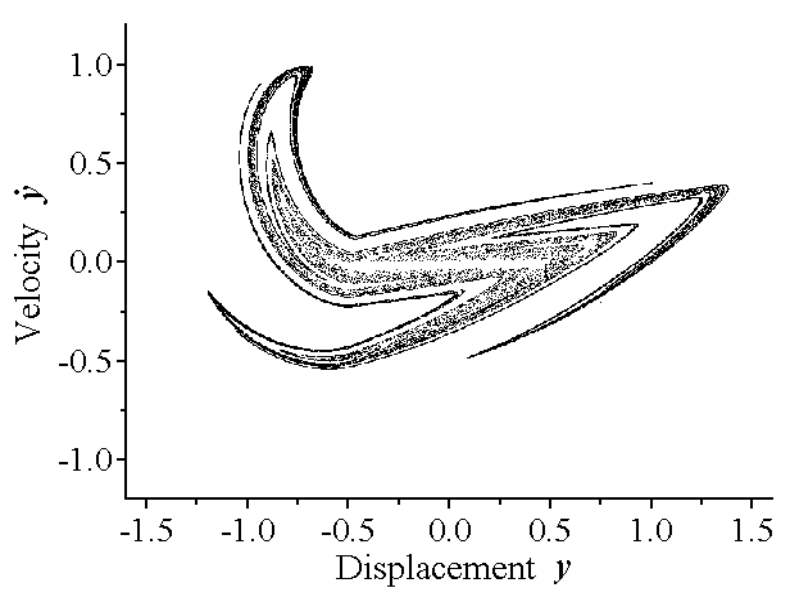

(a) $a=0.01, b=0.3$

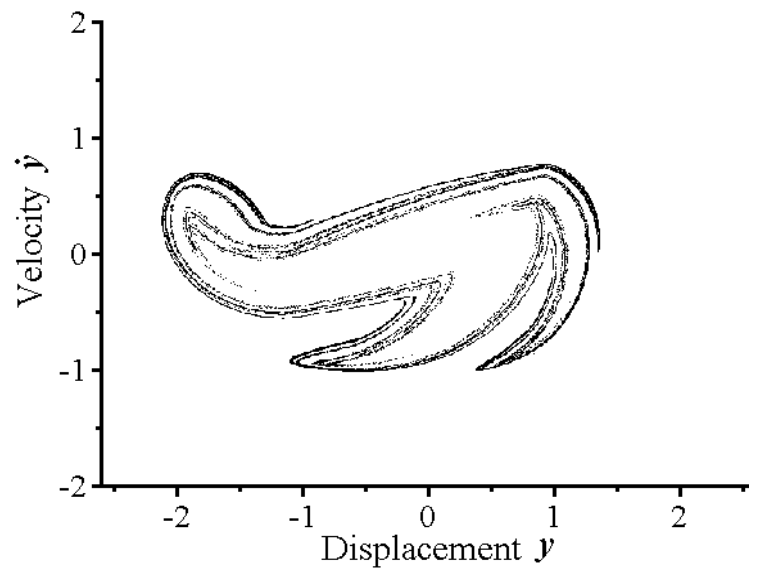

(b) $a=0.17, b=0.283$

Fig. 8. The Poincare sections of the dynamic model with a time-varying clearance at $\varpi=1.1$ and $\gamma=0.5$.

$$
h(\tau, y)=\left\{\begin{array}{r}
(y-1-\gamma \sin \varpi \tau) \\
0 \quad y>1+\gamma \sin \varpi \tau \\
\gamma \sin \varpi \tau-1 \leqslant y \leqslant 1+\gamma \sin \varpi \tau \\
(y+1-\gamma \sin \varpi \tau) \\
y<\gamma \sin \varpi \tau-1
\end{array}\right.
$$

The state of motion of the dynamic system defined by nonlinear equations (19) is determined whenever parameters $a, b, \varpi$ and are specified. For a typical example, however, only $a$ is chosen to vary in the range of 0.01 to 0.6 in the bifurcation and stability of the system, provided that $b=0.3, \varpi=1.1$ and $\gamma=0.5$.

In Fig. 6 are also shown different paths of bifurcation from periodic to chaotic vibrations. Motion of the system is chaotic at the beginning, and suddenly becomes P2 motion. The periodic attractor went through perioddoubling bifurcation to chaos. Being cross critical values, chaotic motion of system changes to P5 oscillation, and further to quasi-period motion at $a=0.42$. Corresponding curve obtained for the largest Lyapunov exponent is given in Fig. 7, by which the existence of positive Lyapunov exponents testifies appearance of chaotic behaviors of the rolling mill.

Investigation to geometrical structures of the strange attractors is made by the Poincare sections. Two diagrams of Poincare sections of the chaotic motions in the rolling mill system with a time-varying clearance are demonstrated respectively in Fig. 8(a) for the parameters $a=0.01, b=0.3, \varpi=1.1$ and $\gamma=0.5$, and in Fig. 8(b) for the parameters $a=0.17, b=0.283$, $\varpi=1.1$ and $\gamma=0.5$.

\section{Concluding remarks}

Asymptotic and numerical analysis of self-excited vibrations of the rolling mill with clearances have been performed according to the simplified dynamic model and the corresponding nonlinear differential equation developed herein. From the viewpoint of real application, the dynamic model is established with consideration of fixed and time-varying clearances. The theoretical and numerical solutions to the nonlinear equation of the dynamic system counting for a fixed clearance by the asymptotic and Runge-Kutta algorithms are made and resembled when the clearance is small enough. This means that the asymptotic method can be employed to construct an effective solution to the nonlinear system with piece-wise restoring force. It has been clearly shown by numerical examples that the self-excited vibration behaviors of the rolling mill system are great affected by the clearances.

It is also found that the bifurcation diagram for the self-excited vibrations of the rolling mill with a timevarying clearance can not be classified into standard period-doubling sequences, but is unique for nonlinear dynamic systems. As observed clearly from the above nonlinear vibrations, a series of states of motion such as periodic, quasi-periodic and chaotic vibrations exist in the nonlinear dynamic model.

The present research may be extended to deal with some nonlinear problems of the self-excited vibration systems. For example, a railway steel bridge that has some clearances in joint supports due to long term wear and tear may vibrate severely in the horizontal plane while the train passes fast through the bridge in the 
S-shaped way. Vibratory screening and conveying machines that would certainly contain clearances and movable boundaries often oscillate seriously and cause big damage. In those nonlinear systems, periodic, quasiperiodic and chaotic vibrations may occur.

\section{References}

[1] N.N. Bogoliubov and Mitropolsk, Asymptotic Methods in the Theory of Nonlinear Oscillation, Gordon and Breach, New York, 1961.

[2] Y.S. Chen, Global Bifurcations and Chaos in a Van Der PolDuffing-Mathieu System with Three-Well Potential Oscillator, Acta Mechanica Sinica 11 (1995), 357-372.

[3] Y.Y. Chen, Analysis of Self-Excited Oscillation in the Slipping State of Blooming Mill, Journal of Northeastern University 3 (1977), 44-53, (in Chinese).

[4] C.W. Gear, Numerical Initial Value Problem in Ordinary Differential Equations, Prentice-Hall Inc., 1971.
[5] J. Guckenhimer and P. Holmes, Nonlinear Oscillations, Dynamical Systems, and Bifurcations of Vector Fields, SpringerVerlag Inc., New York, 1983.

[6] F.C. Moon, J. Cusumano and P.J. Holmes, Evidence For Homoclinic Orbits as A Precursor to Chaos in A Magnetic Pendulum, Physica D 24 (1987), 383-390.

[7] J.M.T. Thompson, A.R. Bokaian and R. Ghaffari, Subharmonic and Chaotic Motions of Compliant Offshore Structures and Articulated Mooring Towers, ASME Journal of Energy Resources Technology 106 (1984), 191-198.

[8] M. Wiercigroch, Chaotic Vibration of a Simple Model of the Machine Tool-Cutting Process System, ASME Journal of Vibration and Acoustics 119 (1997), 468-475.

[9] Y.Y.Xu, The Self-Excited Vibration of System with Clearance, The Proceedings of the 4th National Conference on Vibration, 1986, (in Chinese).

[10] Y.Y. Xu, Self-Excited Vibration with Random Coefficients, ASME Design Engineering Division (Publication) DE 18 (1989), 127-130.

[11] F.L. Zhu, Chaotic Behaviour of a Rub Rotor Model, Journal of Tsinghua University (Science \& Technology) 36 (1996), 52-57, (in Chinese). 

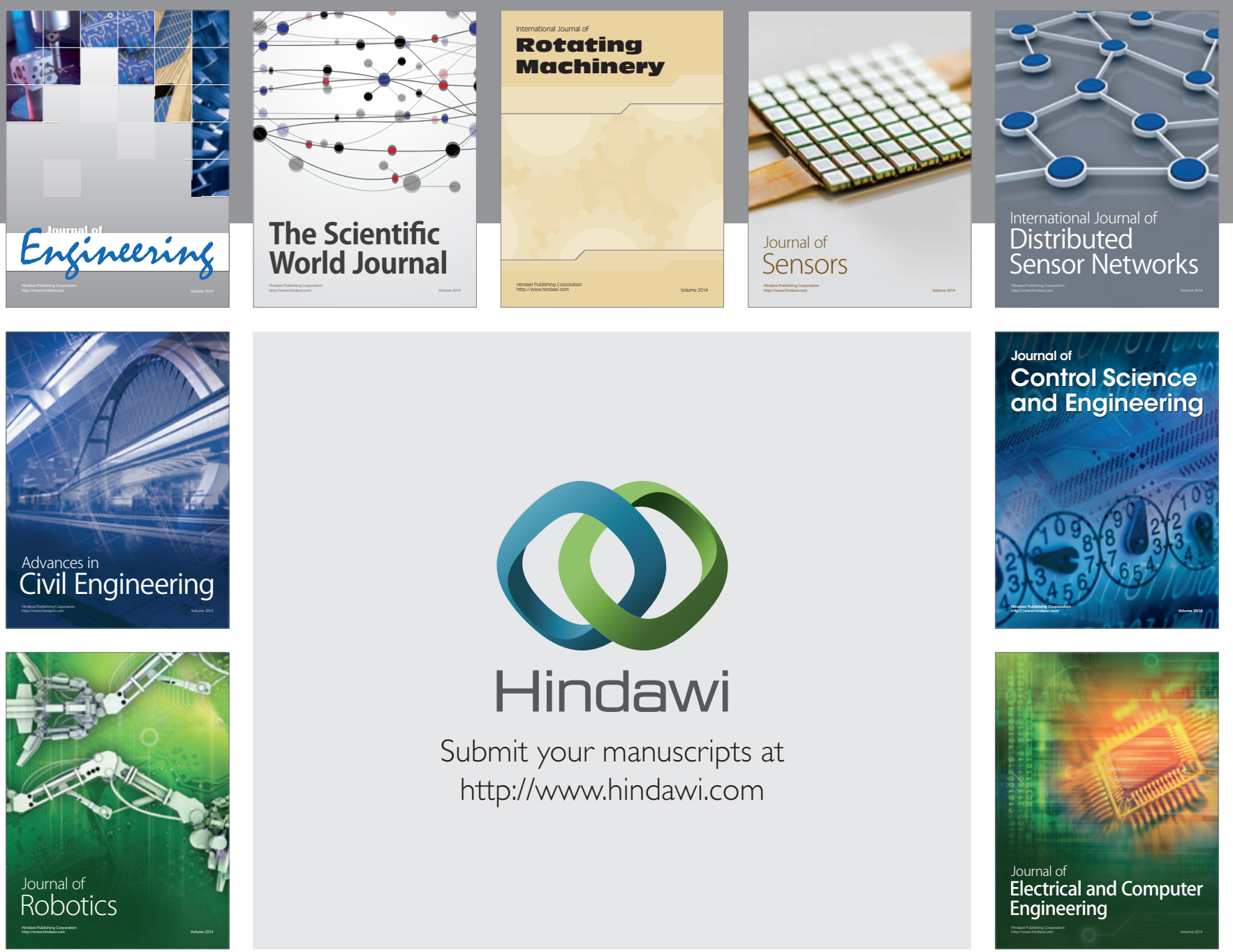

Submit your manuscripts at

http://www.hindawi.com
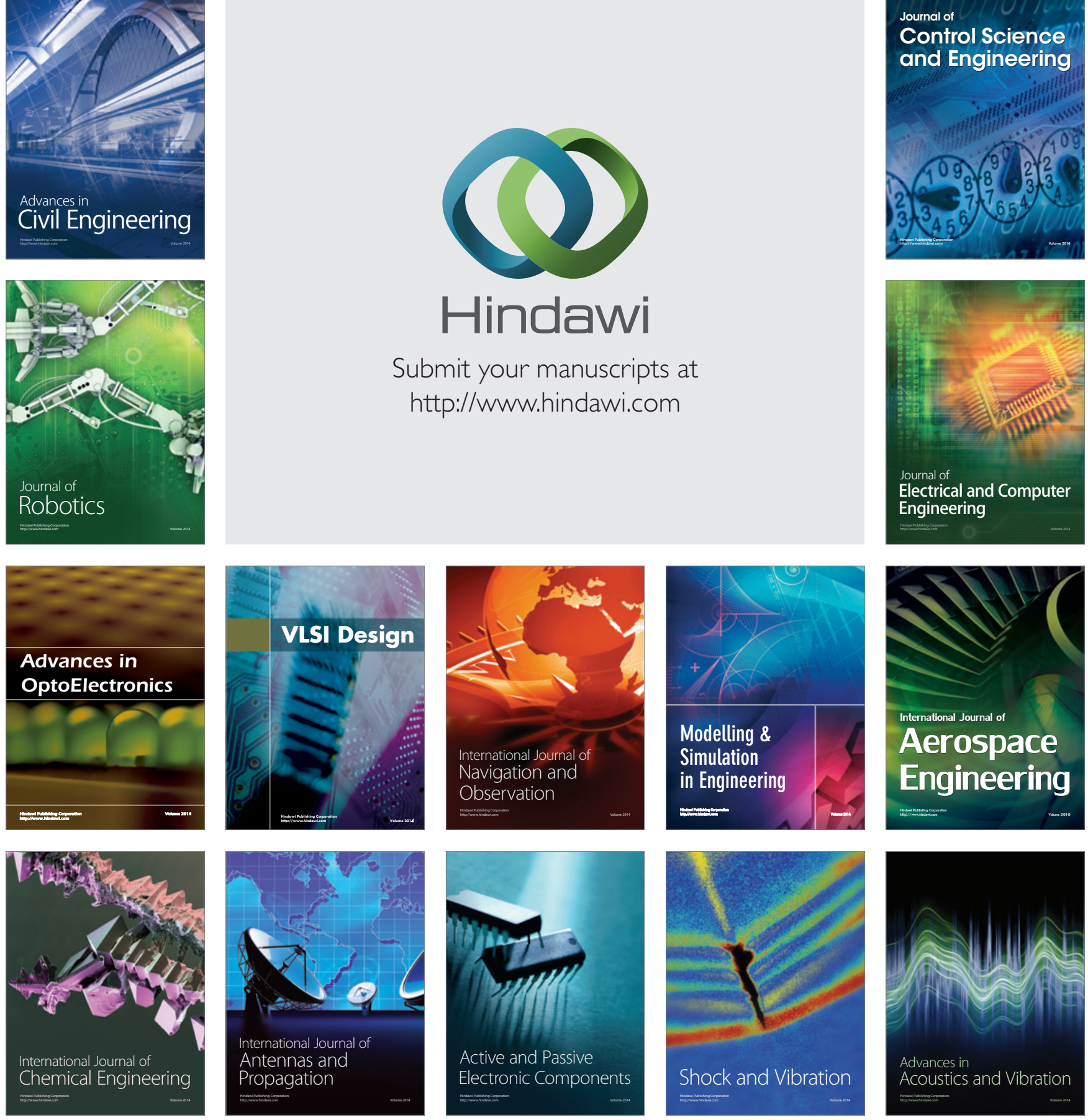